J. Lake Sci. (湖泊科学) , 2019, 31(6): 1749-1757

DOI 10. 18307/2019. 0614

(c) 2019 by Journal of Lake Sciences

\title{
青海湖泉湾镭同位素的空间分布与沿岸地下水排放评价”
}

\author{
苏维刚 ${ }^{1}$, 沙占江 ${ }^{2,4 * *}$, 孔凡翠 ${ }^{3}$, 王求贵 ${ }^{5}$,马茹堂 ${ }^{1}$, 刘 䂞 $^{1}$ \\ (1: 青海省地震局,西宁 810001 ) \\ (2: 青海师范大学生命与地理科学研究院,西宁 810000$)$ \\ (3: 中国科学院青海盐湖研究所,西宁 810000) \\ (4: 青藏高原地表过程与生态保育教育部重点实验室,西宁 810000) \\ (5:核资源与环境国家重点实验室,东华理工大学,南昌 330013)
}

\begin{abstract}
摘 要: 青海湖是青藏高原的重要组成部分, 它是维系青藏高原东北部生态安全的重要水体. 其沿岸地下水的排放直接 影响了青海湖的水文生物地球化学过程, 然而对该问题的研究仍然不充分. 本研究于 2014 年对青海湖泉湾湖水、河水和 地下水进行 ${ }^{223} \mathrm{Ra}$ 和 ${ }^{224} \mathrm{Ra}$ 活度的测定分析, 并对泉湾湖水水平向和垂直向的 Ra 活度分布进行深人分析, 最后估算并探讨 了泉湾水体的停留时间和布哈河口沿岸地下水的排放通量. 表层湖水、河水、地下水不同端元的水体 ${ }^{223} \mathrm{Ra}$ 和 ${ }^{224} \mathrm{Ra}$ 活度值 分别为 0.441 和 $0.026 \mathrm{dpm} / 100 \mathrm{~L} 、 0.22$ 和 $0.016 \mathrm{dpm} / 100 \mathrm{~L}$ 以及 0.061 和 $1.30 \mathrm{dpm} / 100 \mathrm{~L}$, 最高值为地下水, 其次湖水, 河 水最小; 总体上泉湾湖水 ${ }^{223} \mathrm{Ra}$ 和 ${ }^{224} \mathrm{Ra}$ 活度随离岸距离增加分别由 0.047 到 $0.011 \mathrm{dpm} / 100 \mathrm{~L}$ 和 1.4 到 $0.2 \mathrm{dpm} / 100 \mathrm{~L}$ 的 减小,垂向上 Ra 活度呈楔形分布, Ra 活度在不同方向上的非保守性分布主要受到悬浮颗粒物解析的影响; 通过水体滞留 时间模型计算获得青海湖泉湾水体的平均停留时间为 1.8 天,进一步分析泉湾水体 Ra 的源汇项, 应用镭同位素质量平衡 模型获得泉湾布哈河口沿岸地下水排放通量为 $0.054 \sim 0.109 \mathrm{~m}^{3} /\left(\mathrm{m}^{2} \cdot \mathrm{d}\right)$.
\end{abstract}

关键词: 青海湖; 泉湾和布哈河口; ${ }^{223} \mathrm{Ra}$ 和 ${ }^{224} \mathrm{Ra}$ 分布; 地下水排放通量

\section{Evaluation of the groundwater discharge and the spatial distribution of radium isotope in Quanwan Bay of Lake Qinghai}

SU Weigang ${ }^{1}$, SHA Zhanjiang ${ }^{2,4 *}$, KONG Fancui ${ }^{3}$, WANG Qiugui ${ }^{5}$, MA Ruying $^{1} \&$ LIU $^{2}$ Lei $^{1}$

(1: Qinghai Earthquake Agency, Xining 810001, P.R.China)

(2: Institute of Life and Geographical Science, Qinghai Normal University, Xining 810000, P.R.China)

(3: Qinghai Institute of Salt Lakes, Chinese Academy of Sciences, Xining 810000, P.R. China)

(4: MOE Key Laboratory of Tibetan Plateau Land Surface Processes and Ecological Conservation, Xining 810000, P.R. China)

(5: State Key Laboratory of Nuclear Resources and Environment, East China University of Technology, Nanchang 330013, P. R.China)

\footnotetext{
Abstract: Lake Qinghai is an important part of the Qinghai-Tibet Plateau. It is important to maintain the ecological security in the northeast of Qinghai-Tibet Plateau. The discharge of groundwater along the lake shore directly affects the hydrobiogeochemical process of Lake Qinghai. However, research on this issue is still inadequate. In the study, we measured and analyzed the activity of ${ }^{223} \mathrm{Ra}$ and ${ }^{224} \mathrm{Ra}$ in the lake water, river water and groundwater in Quanwan Bay of Lake Qinghai in 2014. We discussed the horizontal and vertical distribution of activity of Ra, and also estimated the residence time of Quanwan Bay water and the discharge flux of groundwater in the Buha Estuary. The activity values of ${ }^{223} \mathrm{Ra}$ and ${ }^{224} \mathrm{Ra}$ at different end elements are $0.441 \mathrm{dpm} / 100 \mathrm{~L}$ and $0.026 \mathrm{dpm} / 100 \mathrm{~L}, 0.22 \mathrm{dpm} / 100 \mathrm{~L}$ and $0.016 \mathrm{dpm} / 100 \mathrm{~L}, 0.061 \mathrm{dpm} / 100 \mathrm{~L}$ and $1.30 \mathrm{dpm} / 100 \mathrm{~L}$, respectively. The activity of Ra from high to low was followed by groundwater, lake water and river water. In general, the activity of ${ }^{223} \mathrm{Ra}$ and ${ }^{224} \mathrm{Ra}$ in Quan-

* 国家自然科学基金项目 (41967053) 资助. 2019-04-23 收稿; 2019-05-14 收修改稿. 苏维刚( 1989 ), 男, 硕士, 工程师; E-mail: yangyuwan_su@163.com.

** 通信作者; E-mail: zhanjiang@ sina.com.
} 
wan Bay were decreased with the increase of offshore distance from 0.047 to $0.011 \mathrm{dpm} / 100 \mathrm{~L}$ and 1.4 to $0.2 \mathrm{dpm} / 100 \mathrm{~L}$, respectively. The activity of Ra was presented wedge in the vertical of lake water. Ra activity in different directions of conservative distribution mainly affected by the suspended particle resolution. The average residence time of Quanwan Bay water was calculated by using the water retention time model, and the source and sink term of Ra in Quanwan Bay was further analyzed. The discharge flux of groundwater was $0.054-0.109 \mathrm{~m}^{3} /\left(\mathrm{m}^{2} \cdot \mathrm{d}\right)$ in the Buha Estuary which was calculated by the radium isotope mass balance model.

Keywords: Lake Qinghai; Quanwan Bay; Buha Estuary; distribution of ${ }^{223}$ Ra and ${ }^{224} \mathrm{Ra}$; discharge flux of groundwater

${ }^{223,224,226,228} \mathrm{Ra}$ 作为天然放射性同位素,主要由铀系和钍系衰变产生. 由于镭同位素的半颗粒活性,在淡 水环境中 $\mathrm{Ra}$ 表现为颗粒物活性, 而在咸水环境中, 可以与其他电荷和半径相似的离子竞争而从颗粒物中解 析进人水体 ${ }^{[1-3]}$. ${ }^{223} \mathrm{Ra}$ 和 ${ }^{224} \mathrm{Ra}$ 半衰期分别是 11.4 和 $3.66 \mathrm{~d}$, 分别为研究 $10 \sim 50$ 和 $1 \sim 10 \mathrm{~d}$ 的近岸海域水体循 环与混合的理想示踪剂 ${ }^{[4-7]} .1996$ 年 Moore 在美国东海岸推测水体中 ${ }^{226} \mathrm{Ra}$ 存在着一重要输人源为海底地下 水排放 ( submarine groundwater discharge, SGD ${ }^{[8]}$, 并由此拉开了镭同位素示踪技术对 SGD 研究的序幕. 在 海湾、河口、沼泽以及泻湖等环境中应用镭同位素成为评价 SGD 最常用的地球化学示踪剂 ${ }^{[9-14]}$.

青海湖位于青藏高原东北缘, 是我国最大的内陆封闭性咸水湖, 为青藏高原的重要组成部分, 它是维系 青藏高原东北部生态安全的重要水体, 同时也是控制西部荒漠化向东曼延的天然屏障 ${ }^{[15]}$, 是我国西北干旱 区、西南高寒区和东部季风区的交汇区域,对气候变化异常敏感 ${ }^{[16]}$, 因此针对青海湖及其流域的研究具有非 常重要的科学价值和实际意义. 近些年由于全球气候和人类活动变化, 使青海湖流域水位和水质存在一系 列的问题 ${ }^{[17-18]}$, 因而已有报道通过水量平衡模型针对青海湖以及子湖尔海、耳海进行了大量研究,包括对降 水量、蒸发量、地表径流、地下径流、融雪、冰川融水等因子的比较研究, 分别估算了青海湖的降水量、蒸发 量、地表径流量来评估青海湖地下水的输人量, 并进而解释了青海湖水位的盈亏状况以及预测未来水位的 变化 ${ }^{[19-21]}$. 然而这种结果得到的地下水排放通量较为粗略, 不能详细地获得地下水排放通量及对湖泊生物 地球化学过程的影响. 沙占江等分别利用 ${ }^{226} \mathrm{Ra} 、{ }^{228} \mathrm{Ra}$ 和 ${ }^{223} \mathrm{Ra} 、{ }^{224} \mathrm{Ra}$ 计算获得了青海湖子湖尔海的地下水排 放通量 ${ }^{[22-23]}$, Kong 等应用镭同位素示踪方法对青海湖西北岸的水域进行了生物地球化学过程方面的研 究 $^{[24-26]}$, 但针对青海湖湖区地下水排放的示踪研究尚未有更多研究资料. 因此本文尝试对青海湖泉湾的河 流水、地下水、湖水中的 ${ }^{223} \mathrm{Ra}$ 和 ${ }^{224} \mathrm{Ra}$ 分布进行分析来计算相应湖区的地下水排放通量, 从而深人了解青海 湖湖泊生物地球化学进程.

\section{1 区域地质背景}

青海湖 (36 $32^{\prime} \sim 37^{\circ} 15^{\prime} \mathrm{N}, 99^{\circ} 36^{\prime} \sim 100^{\circ} 16^{\prime} \mathrm{E}$ ) 位于南祁连早古生代裂陷槽、青海南山晚古生代一中生代 复合裂陷槽和中祁连地块这 3 个构造单元的交汇部位, 区内主要有三条大的断裂带 ${ }^{[27]}$. 自中中新世开始随 新构造运动的作用青海湖的形成演化经历了四个主要时期, 分别为构造洼地、地堑、河湖并存以及全盛期, 而随着气候变化青海湖多次发生波动性退缩, 青海湖现代湖泊演变规律是受新构造运动及气候因素综合作 用的影响 ${ }^{[28-29]}$. 青海湖是中国最大的内陆湖泊, 也是中国最大的咸水湖, 面积达 $4351.50 \mathrm{~km}^{2}$, 环湖周长 360 $\mathrm{km}$, 湖面东西长、南北窄. 青海湖湖水平均深约 $19 \mathrm{~m}$ 多, 青海湖湖水补给来源主要是河水, 其次是湖底的泉 水和降水. 湖周大小河流有 70 余条, 呈明显的不对称分布. 布哈河是流人湖中最大的一条河, 年径流量 11.2 亿 $\mathrm{m}^{3}$, 占人湖径流 $60 \%$. 青海湖每年获得径流补给主要是布哈河、沙柳河、泉吉河和哈尔盖河,这 4 条大河的 年径流量达 16.12 亿 $\mathrm{m}^{3}$, 占人湖径流量的 $86 \%$, 河流径流年内分配不均, 6-9 月径流量约占全年径流量的 $75 \%$ 以上. 青海湖具有高原大陆性气候, 年均温在 $1.1 \sim 0.3^{\circ} \mathrm{C}$ 之间, 年均降雨量在 $319 \sim 395 \mathrm{~mm}$ 之间, 降雨主 要集中在 6-9 月, 约占全年降雨量的 $80 \%$. 青海湖泉湾位于青海湖西部, 为一 $\mathrm{U}$ 型湖湾, 湖湾面积约 $246.998 \mathrm{~km}^{2}$, 湖湾平均水深约 $12 \mathrm{~m}$, 湖湾有布哈河注人.

\section{2 实验方法}

样品于 2014 年采自青海湖西北岸, 总计 26 个. 湖水样品 23 个 (图 1), 其中包括 16 个表层水体样品和 7 个垂向深度的样品, 表层水体样品采自距离湖面 $1 \mathrm{~m}$ 的深度位置, 垂向深度样品采自距湖面 $6 \sim 18 \mathrm{~m}$ 的不同 深度位置. 此外采集了 2 个地下水样品和 2 个河水样品, 河水样品从人湖口开始沿河流间隔 $1 \sim 2 \mathrm{~km}$ 采取. 
每个样点采集水量 $50 \mathrm{~L}$, 样品数据见表 1 和表 2 .

现场使用便携式多参数水质分析仪 (型号 EC300; 美国 YSI 生产) 通过电导法测定获取每个样点的盐 度、 $\mathrm{pH}$ 值、溶解氧 $(\mathrm{DO})$ 、温度等参数, 之后将采集的水样通过镇纤维单柱富集法进行富集 ${ }^{[30]}$, 富集柱装填 $15 \mathrm{~g}$ 锰纤维, 在富集柱前面接人 $1 \mu \mathrm{m}$ 的过滤桶, 富集时, 水流速控制在 $450 \sim 550 \mathrm{ml} / \mathrm{min}$, 后将锰纤维的含水 率控制在 $1.5^{[31]}$, 并通过 RaDeCC 进行 ${ }^{223} \mathrm{Ra}$ 和 ${ }^{224} \mathrm{Ra}$ 的比活度测试, 该仪器为美国 Scientific Computer Instruments 公司生产的四通道同步延时计数器.

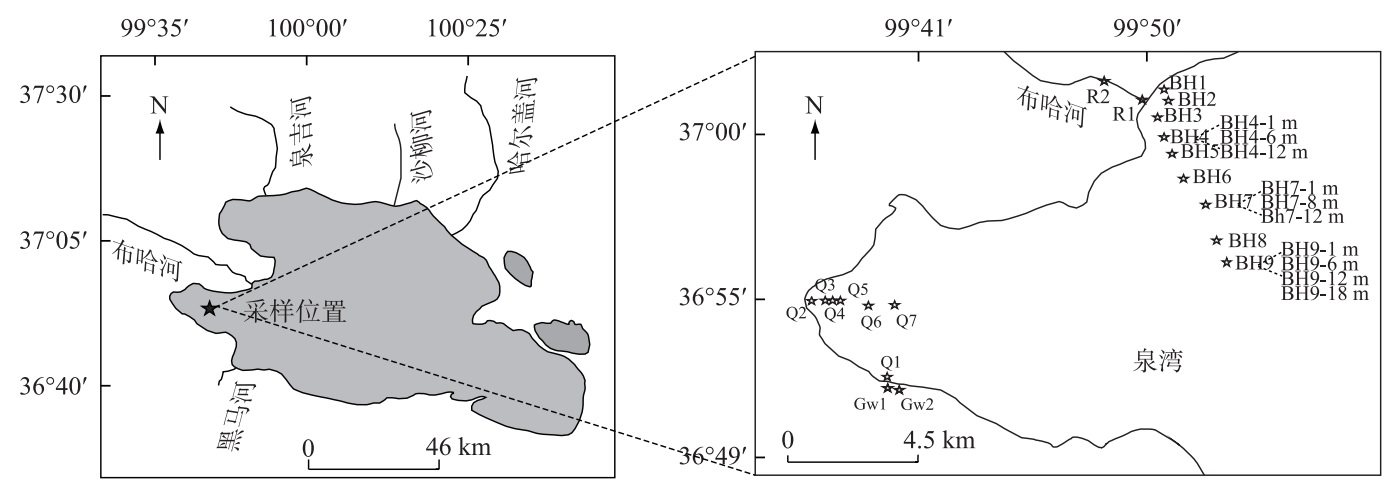

图 12014 年青海湖泉湾采样位置

Fig.1 Sampling location of Quanwan Bay in Lake Qinghai in 2014

表 1 湖水、河水和地下水镭活度及各种参数

Tab.1 Ra activities and different parameters in lake, river and groundwater

\begin{tabular}{lccccccccc}
\hline 样品 & & $\begin{array}{c}\text { 离岸距离/ } \\
\mathrm{km}\end{array}$ & $\begin{array}{c}\text { 温度/ } \\
{ }^{\circ} \mathrm{C}\end{array}$ & $\begin{array}{c}\text { 盐度/ } \\
(\mathrm{g} / \mathrm{L})\end{array}$ & $\begin{array}{c}\mathrm{DO} / \\
(\mathrm{mg} / \mathrm{L})\end{array}$ & $\mathrm{pH}$ & $\begin{array}{c}{ }^{224} \mathrm{Ra} / \\
(\mathrm{dpm} / 100 \mathrm{~L})\end{array}$ & $\begin{array}{c}{ }^{223} \mathrm{Ra} / \\
(\mathrm{dpm} / 100 \mathrm{~L})\end{array}$ & $\begin{array}{c}{ }^{224} \mathrm{Ra} /{ }^{223} \mathrm{Ra} \\
\mathrm{AR}\end{array}$ \\
\hline 表层湖水 & $\mathrm{BH} 1 \sim \mathrm{BH} 9$ & $0.84 \sim 7.65$ & $11.8 \sim 16.1$ & $2.5 \sim 11.3$ & $0.25 \sim 0.29$ & $9.26 \sim 9.438$ & $0.2 \sim 1.4$ & $0.011 \sim 0.047$ & $5.13 \sim 29.79$ \\
& $\mathrm{Q} 1 \sim \mathrm{Q} 7$ & $0.035 \sim 2.81$ & $10.0 \sim 15.2$ & $8.8 \sim 10.6$ & $0.22 \sim 0.32$ & $10.005 \sim 10.45$ & $0.15 \sim 0.52$ & $0.005 \sim 0.068$ & $7.65 \sim 88.00$ \\
河水 & $\mathrm{R} 1 \sim \mathrm{R} 2$ & - & - & 0.2 & - & - & $0.20 \sim 0.24$ & $0.011 \sim 0.020$ & - \\
地下水 & $\mathrm{Gw} 1 \sim \mathrm{Gw} 2$ & - & 10.0 & 0.3 & 0.14 & 9.598 & $1.26 \sim 1.34$ & $0.048 \sim 0.073$ & - \\
\hline
\end{tabular}

一表示数据缺失.

表 2 不同深度的湖水镭活度

Tab.2 Ra activities of lake water in different depths

\begin{tabular}{cccccccc}
\hline 样品 & 深度 $/ \mathrm{m}$ & 温度 $/{ }^{\circ} \mathrm{C}$ & 盐度 $/(\mathrm{g} / \mathrm{L})$ & $\mathrm{DO} /(\mathrm{mg} / \mathrm{L})$ & $\mathrm{pH}$ & ${ }^{224} \mathrm{Ra} /(\mathrm{dpm} / 100 \mathrm{~L})$ & ${ }^{223} \mathrm{Ra} /(\mathrm{dpm} / 100 \mathrm{~L})$ \\
\hline BH4 & $1 \sim 12$ & $14.9 \sim 15.8$ & $11.0 \sim 11.5$ & $0.24 \sim 0.28$ & $9.192 \sim 9.287$ & $0.18 \sim 0.32$ & 0.01 \\
BH7 & $1 \sim 12$ & $15.8 \sim 16.1$ & $11.0 \sim 11.8$ & $0.23 \sim 0.28$ & $9.298 \sim 9.368$ & $0.15 \sim 0.31$ & $0.006 \sim 0.014$ \\
BH9 & $1 \sim 18$ & $15.0 \sim 15.4$ & $10.3 \sim 11.9$ & $0.23 \sim 0.29$ & $9.404 \sim 9.434$ & $0.12 \sim 0.29$ & $0.001 \sim 0.018$ \\
\hline
\end{tabular}

\section{3 结果与讨论}

湖水、地下水和河水的 ${ }^{223} \mathrm{Ra}$ 和 ${ }^{224} \mathrm{Ra}$ 活度结果见表 1 和表 2 , 地下水 Ra 活度最高, 其次为湖水 $\mathrm{Ra}$ 活度, 表层湖水的 Ra 活度整体略高于河水 Ra 活度 (图 2), 且湖水 $R a$ 活度分布区间较大, 其中 Q2 点 $(d=0.2 \mathrm{~km})$ 的 ${ }^{223} \mathrm{Ra}$ 和 ${ }^{224} \mathrm{Ra}$ 活度比值最高为 $88,{ }^{223} \mathrm{Ra}$ 和 ${ }^{224} \mathrm{Ra}$ 活度分别为 0.005 和 $0.44 \mathrm{dpm} / 100 \mathrm{~L}$.

泉湾区域镭的源项主要为地下水和河水悬浮颗粒物解析, 镭的汇项主要为镭的自身放射性衰变和与湖 水的混合. 在地下水向湖水输人过程中, 镭同位素活度由于自身衰变和与湖水混合将不断降低, 因此在地下 
水、河水、湖水三个水体端元中, 地下水 Ra 活度最高, 湖水 Ra 活度次之, 河水 Ra 活度最低. 地下水的流速比 地表水要小好几个数量级, 在含水层运移中水一岩间充分相互作用, 从而造成地下水中 $\mathrm{Ra}$ 含量普遍较河流 等地表水体要高很多,而湖水 Ra 活度受悬浮颗粒物解析等影响产生较大的变化区间 ${ }^{[1,32-34]}$.
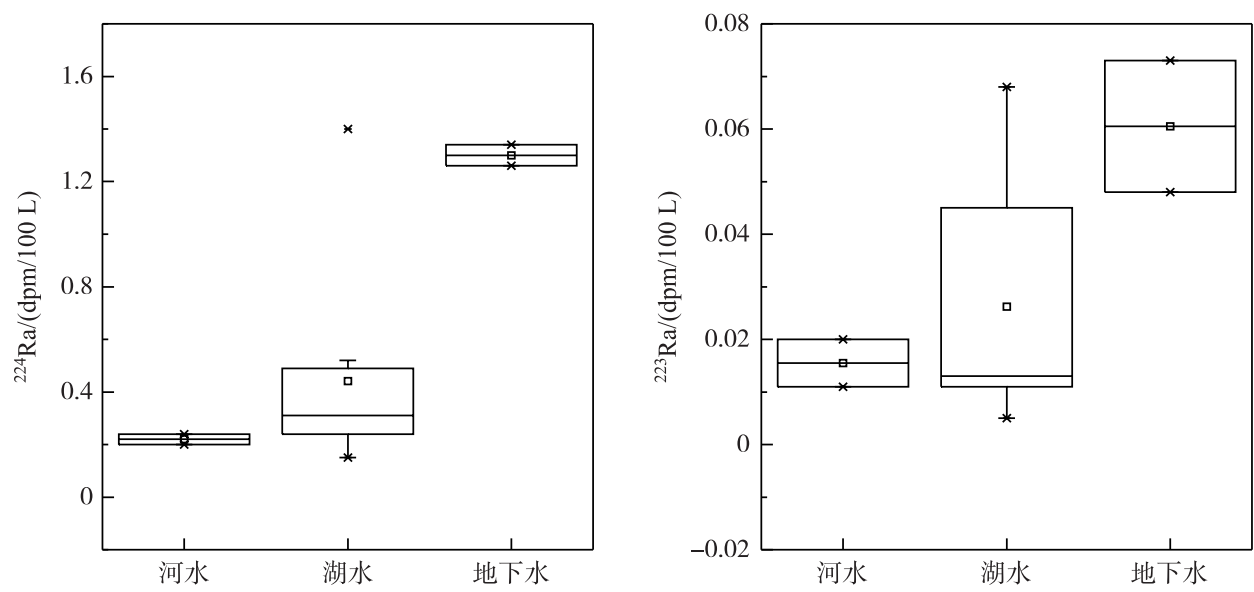

图 2 不同端元水体中的 ${ }^{223} \mathrm{Ra}$ 和 ${ }^{224} \mathrm{Ra}$ 活度对比

Fig. 2 Comparison of ${ }^{223} \mathrm{Ra}$ and ${ }^{224} \mathrm{Ra}$ activities in different end-element waters

\section{1 湖水中的镭同位素空间分布特征}

${ }^{223} \mathrm{Ra}$ 和 ${ }^{224} \mathrm{Ra}$ 活度在湖水表层的分布如图 3 和图 4 所示, 其中泉湾 $\mathrm{Ra}$ 活度随离岸距离增加而减小, 布 哈河口 Ra 活度随离岸距离增加呈先减小后增大再急剧减小的趋势, Ra 活度增大的高值点 (BH2) 离岸 1.4 $\mathrm{km}$ 左右, Ra 活度随离岸距离的增加呈减小趋势,但在某一区间内又略微增大的非保守性分布.
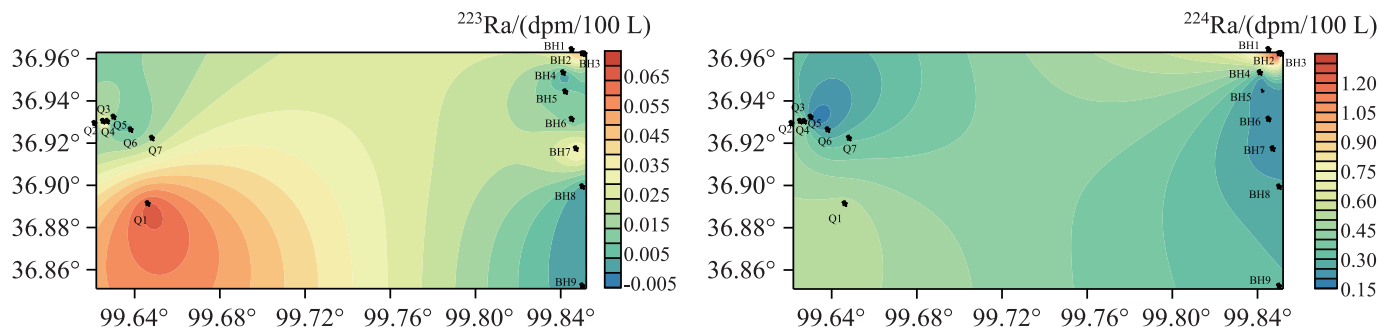

图 3 泉湾湖水的 ${ }^{223} \mathrm{Ra}$ 和 ${ }^{224} \mathrm{Ra}$ 活度随离岸距离的空间分布

Fig. 3 The spatial distribution of ${ }^{223} \mathrm{Ra}$ and ${ }^{224} \mathrm{Ra}$ activities of lake water with offshore distance in Quanwan Bay

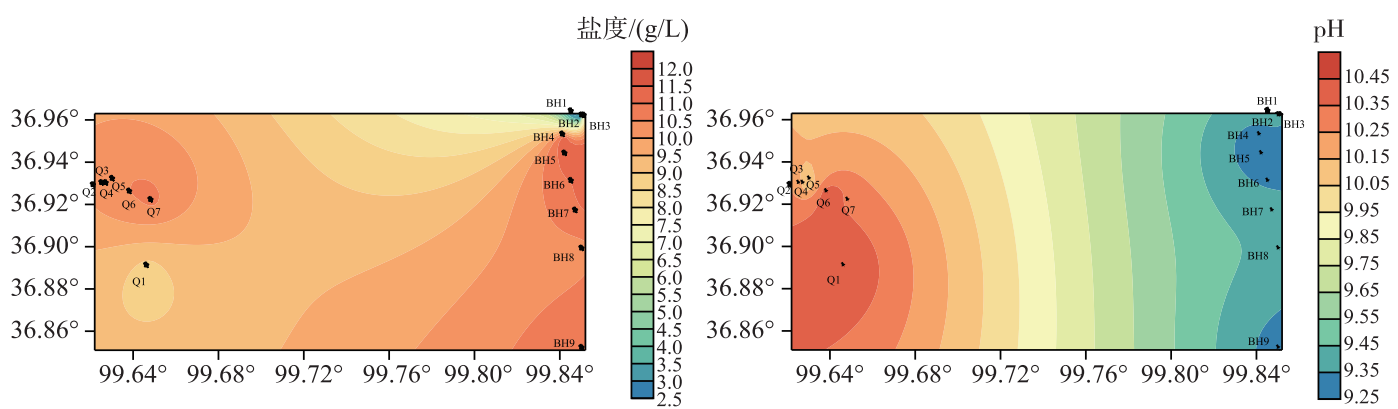

图 4 泉湾湖水的盐度和 $\mathrm{pH}$ 随离岸距离的空间分布

Fig.4 The spatial distribution of salinity and $\mathrm{pH}$ of lake water with offshore distance in Quanwan Bay 
在地表淡水中, ${ }^{223} \mathrm{Ra}$ 和 ${ }^{224} \mathrm{Ra}$ 通常吸附在悬浮颗 粒上, 在混合区盐度增加, ${ }^{223} \mathrm{Ra}$ 和 ${ }^{224} \mathrm{Ra}$ 从固体颗粒 表面解析下来而以溶解态形式存在, 且湖水盐度越 高, 解析得越彻底 ${ }^{[2-3,35]}$, 结合湖水 Ra 活度的表层分 布和盐度分布来看 (图 3, 图 4), 布哈河口盐度随离 岸距离的增加而增大, 在 BH8 开始略微减小, 湖水表 层的盐度分布与 Ra 活度的分布具有一定的相似性, 而布哈河口的 $\mathrm{pH}$ 整体低于泉湾的 $\mathrm{pH}$ 值, 且随离岸 距离的增大没有明显的线性分布特征, 布哈河口表层 湖水的 Ra 活度呈非保守性分布, 表明表层湖水中 Ra 活度受到盐度对悬浮颗粒物解析程度的影响. 如图 5 所示, 在布哈河口混合区, 由于悬浮颗粒物的解析作

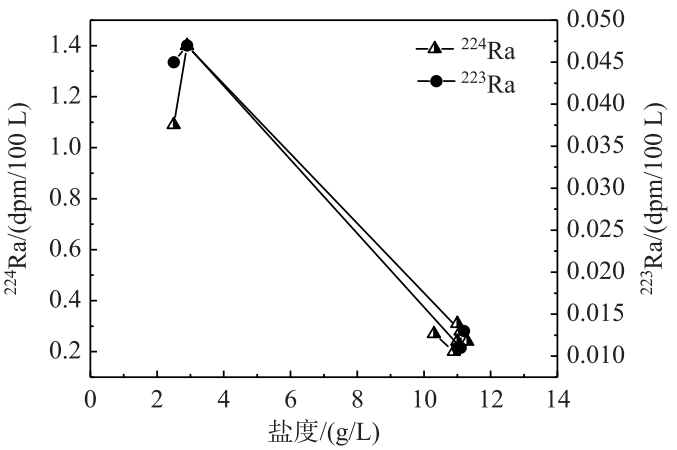

图 $5 \mathrm{Ra}$ 活度随盐度的相关变化

Fig.5 The Ra activity changes with salinity 用, 水体中溶解的 Ra 活度会随盐度的增加而增加. 当盐度增加到 $2.9 \mathrm{~g} / \mathrm{L}(\mathrm{BH} 2)$, Ra 活度达到最大 $\left({ }^{224} \mathrm{Ra}=\right.$ $1.4 \mathrm{dpm} / 100 \mathrm{~L}$ 和 ${ }^{223} \mathrm{Ra}=0.047 \mathrm{dpm} / 100 \mathrm{~L}$ ). 之后随着盐度的增加, ${ }^{224} \mathrm{Ra}$ 和 ${ }^{223} \mathrm{Ra}$ 活度迅速下降, 说明解析已 经完成. 前人研究发现盐度在 $10 \sim 11 \mathrm{~g} / \mathrm{L}$ 时, Ra 同位素的解析量达到最大值 ${ }^{[6,35-39]}$. 然而本次研究得到的结 果与其具有不一致, $\mathrm{Ra}$ 的解析受控因素有多种, 诸如悬浮颗粒物含量 (SPM)、水体盐度、 $\mathrm{pH}$ 、温度等 等 ${ }^{[6,34,40]}$. Jin 等通过对布哈河口悬浮颗粒物化学组分的输人通量研究, 发现包括 $\mathrm{SiO}_{2} 、 \mathrm{Al}_{2} \mathrm{O}_{3} 、 \mathrm{Fe}_{2} \mathrm{O}_{3} 、 \mathrm{MgO} 、$ $\mathrm{K}_{2} \mathrm{O} 、 \mathrm{TiO}_{2}$ 等颗粒物组分在河流人湖口最高, 随离岸距离增大而减小 ${ }^{[41]}$, 在本次研究中 $\mathrm{BH} 3(\mathrm{~d}=1.41 \mathrm{~km})$ 的 离岸距离大于 $\mathrm{BH} 2(\mathrm{~d}=1.37 \mathrm{~km})$, 颗粒物的含量和稀释过程导致了 BH3(盐度 $11.1 \mathrm{~g} / \mathrm{L}$ ) 中的 Ra 同位素的 活度值要比 $\mathrm{BH} 2$ (盐度 $2.9 \mathrm{~g} / \mathrm{L}$ ) 的要低.

在垂向分布上 (图 6), 表层湖水的 $\mathrm{Ra}$ 活度高于深部湖水的 $\mathrm{Ra}$ 活度, ${ }^{224} \mathrm{Ra}$ 和 ${ }^{223} \mathrm{Ra}$ 活度随湖水深度增加 大体呈减小趋势, 说明主要受 Ra 的放射性衰变和湖水混合影响, 但在一定深度存在小幅增大的非保守性分 布, 且随着湖岸向湖心分布, 非保守性分布的样点深度逐渐增大.
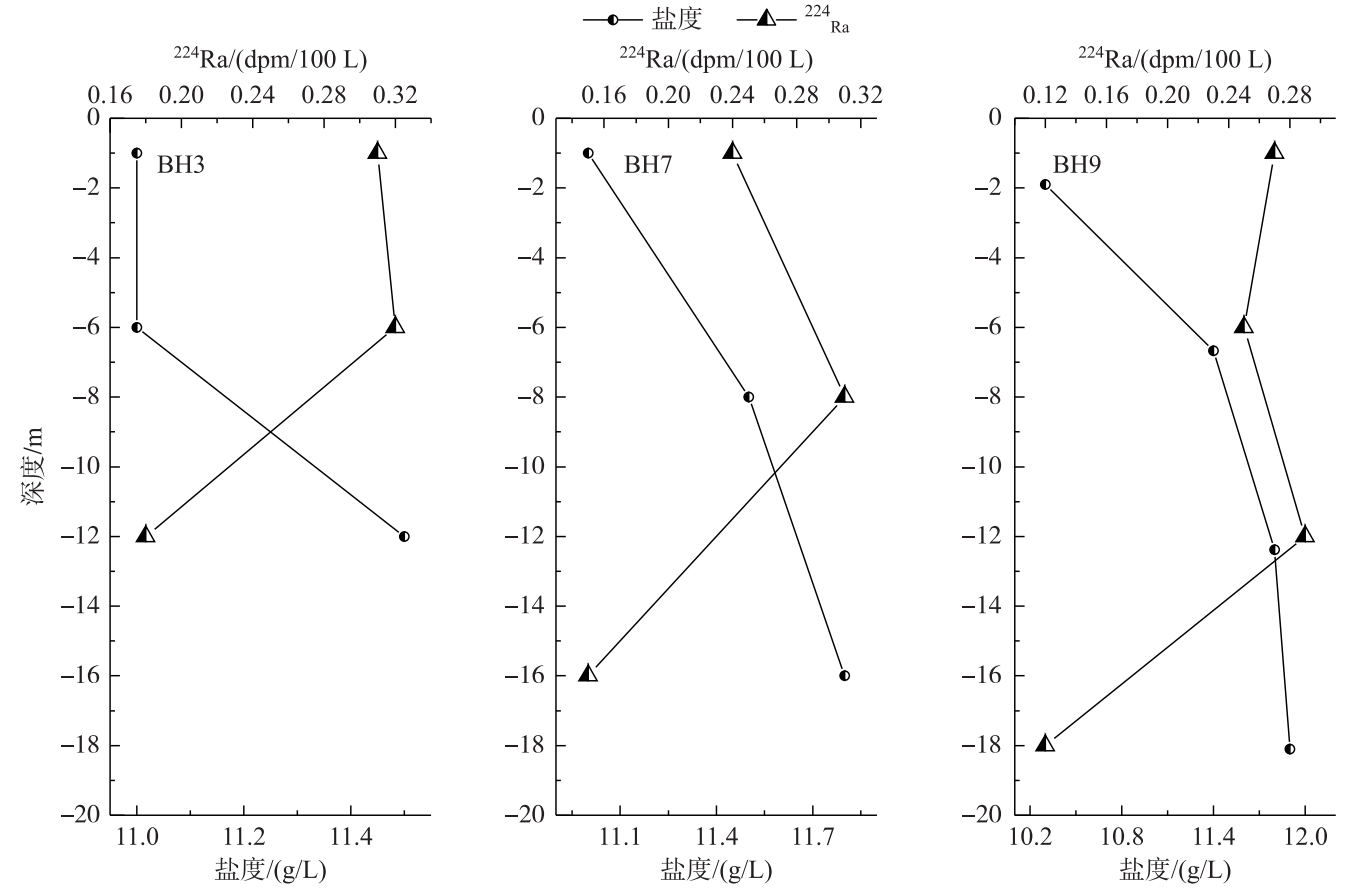

图 6 镭活度随深度的相关变化

Fig. $6{ }^{224}$ Ra activity and salinity changes with depth 
由于湖水为咸水, 地下水和河水为淡水, 湖水密度大于河水和地下水, 因此, 在河口的混合过程中, 输人 的河水和沿岸地下水由于密度小的原因会先在上层, 而湖水在底层, 从而导致在一定深度内 Ra 的解析活度 达到最大值. 而底部由于混合稀释和快速衰变的原因导致其 Ra 活度低于其最高值, 从而形成了楔形的剖面 分布 ${ }^{[1,8]}$, 这和前人在海洋河口得到的垂向分布有所不同, 海洋河口底部镭活性较高, 主要来源于底部沉积 物的扩散, 也有可能有海底地下水的输送 ${ }^{[3,5,42-43]}$. 在样点 $\mathrm{BH} 3(\mathrm{~d}=1.41 \mathrm{~km})$ Ra 活度在水深 $6 \mathrm{~m}$ 处达到最大 值; 在样点 $\mathrm{BH} 7(\mathrm{~d}=4.1 \mathrm{~km})$ Ra 活度在水深 $8 \mathrm{~m}$ 处达到最大值; 在样点 $\mathrm{BH} 9(\mathrm{~d}=7.65 \mathrm{~km}) \mathrm{Ra}$ 活度先减小, 然 后在在水深 $12 \mathrm{~m}$ 处达到最大值, 水体混合的最大解析面 ${ }^{224} \mathrm{Ra}$ 和 ${ }^{223} \mathrm{Ra}$ 活度的范围分别为 $0.29 \sim 0.32$ 和 $0.014 \sim 0.018 \mathrm{dpm} / 100 \mathrm{~L}$.

表 3 青海湖水体停留时间

Tab.3 Water residence time in the Lake Qinghai

\begin{tabular}{cccc}
\hline 样品 & $A R$ & $\begin{array}{c}\text { 停留时间/ } \\
\mathrm{d}\end{array}$ & $\begin{array}{c}\text { 离岸距离/ } \\
\mathrm{km}\end{array}$ \\
\hline $\mathrm{BH} 2$ & 29.79 & 1.21 & 1.37 \\
$\mathrm{BH} 3$ & 25.45 & 1.37 & 1.41 \\
$\mathrm{BH} 7$ & 21.82 & 1.52 & 4.10 \\
$\mathrm{BH} 8$ & 5.13 & 2.97 & 5.70 \\
Q3 & 9.07 & 2.40 & 0.62 \\
Q4 & 12.50 & 2.08 & 0.71 \\
Q6 & 26.00 & 1.35 & 1.86 \\
Q7 & 16.67 & 1.79 & 2.81 \\
\hline
\end{tabular}

\section{2 水体停留时间}

Moore 在对南大西洋湾陆架淡水锋的研究中提出了一种模 型, 利用 ${ }^{224} \mathrm{Ra} /{ }^{223} \mathrm{Ra}$ 来估计大陆架水域的停留时间, 即镭同位素 从镭源进人水体后跟随水体所经历的运移时间.

$$
A R_{\text {obs }}=A R_{\mathrm{i}} \frac{\mathrm{e}^{-\lambda_{224} t}}{\mathrm{e}^{-\lambda_{22} t}}
$$

式中, $\lambda_{223}$ 的衰变常数为 $0.06 \mathrm{~s}^{-1}, \lambda_{224}$ 的衰变常数为 $0.19 \mathrm{~s}^{-1}, A R_{\mathrm{i}}$ 是 ${ }^{224} \mathrm{Ra} /{ }^{223} \mathrm{Ra}$ 的初始活度比, $A R_{\text {obs }}$ 是样品中 ${ }^{224} \mathrm{Ra} /{ }^{223} \mathrm{Ra}$ 的比值. 该方法假设初始的 ${ }^{224} \mathrm{Ra} /{ }^{223} \mathrm{Ra}$ 活度比 (activity ratio, $A R$ ) 是恒定 的, 只有当湖水与镭源分离时才会发生衰变 ${ }^{[44]}$.

在研究中镭源点通常选择最高的镭同位素比值 ${ }^{[45]}$. 由于 $\mathrm{Q} 2$ 的值最高, 并且位于河口区, 因此我们选择选择 Q2 作为 $A R_{\mathrm{i}}$. 通过计算得到了水体停留时间 (表 3 ). 青海湖泉湾水体的平均 停留时间为 1.8 天.

\section{3 布哈河口沿岸地下水排放 ( Lake Groundwater Discharge,简称 LGD)}

${ }^{223} \mathrm{Ra}$ 和 ${ }^{224} \mathrm{Ra}$ 都来自于颗粒物上具有颗粒活性的 $\mathrm{Th}$ 的放射性衰变. 在淡水中镭具有颗粒活性, 吸附在 颗粒物上, 遇到咸水由于其他阳离子与其进行竞争, 通过离子交换作用从颗粒物上解析到水体中. 布哈河口 源项有河流输人、颗粒物解析、沉积物扩散和地下水输人, 孔凡翠等通过室内模拟湖底沉积物扩散的 ${ }^{226} \mathrm{Ra}$ 和 ${ }^{228} \mathrm{Ra}$ 的量分别为 0.018 和 $0.092 \mathrm{dpm} /\left(\mathrm{m}^{2} \cdot \mathrm{h}\right),{ }^{223} \mathrm{Ra}$ 和 ${ }^{224} \mathrm{Ra}$ 的量低于检测限 ${ }^{[39]}$. 在大湖深湖中由于其深 度的影响 (超过 $10 \mathrm{~m}$ ), 湖水可以分成 3 层, 其底层水在 7 月为稳定水层, 因此在该季节沉积物向水体中扩散 的 ${ }^{223} \mathrm{Ra}$ 和 ${ }^{224} \mathrm{Ra}$ 在水体中主要为自由扩散, 由于 ${ }^{223} \mathrm{Ra}$ 和 ${ }^{224} \mathrm{Ra}$ 短的半衰期, 导致其往顶部扩散过程衰变殆尽, 因此湖底沉积物扩散量可以忽略; 汇项有放射性衰变和与湖水混合. 明确 ${ }^{224} \mathrm{Ra}$ 的源汇项之后, 利用相关参数 （表 4)，经过计算得到 ${ }^{224} \mathrm{Ra}$ 源、汇的各个数值.

布哈河口溶解态的 $224 \mathrm{Ra}$ 总量为 $2.13 \times 10^{11} \mathrm{dpm} / \mathrm{a}$, 主要由地下水输人和悬浮颗粒物解析提供, 而河流输 人不到总量的 $1 \%$ (表 5). 利用地下会端元的 ${ }^{224} \mathrm{Ra}$ 活度经 $\mathrm{F}_{\mathrm{LGD}}=\mathrm{I}_{\mathrm{LGD}} / \mathrm{A}_{\mathrm{GW}}$ 计算得出布哈河口沿岸表层地下水 平均每平方米的排放通量为 $0.54 \mathrm{~m}^{3} /\left(\mathrm{m}^{2} \cdot \mathrm{d}\right)$. 根据 SGD 中再循环海水和地下淡水的论述可知, 通过镭同位 素质量模型获得的地下水排放通量也包括再循环的湖水, 因为湖水水动力较海水而言非常弱, 因此我们参 照 SGD 中地下水所占 $10 \% \sim 30 \%$ 的比例选取 10\% 20\%作为湖泊地下水的排放通量 ${ }^{[1,46-48]}$, 则青海湖泉湾布 哈河口平均每平米地下水排放通量为 $0.054 \sim 0.109 \mathrm{~m}^{3} /\left(\mathrm{m}^{2} \cdot \mathrm{d}\right)$.

统计了近年来针对青海湖沿岸地下水排放通量的研究结果, 通过表 6 可以看出, 本文通过镭同位素示 踪的 $\mathrm{F}_{\mathrm{LGD}}$ 与近些年有关学者通过水量平衡模型计算出的 $\mathrm{F}_{\mathrm{LGD}}$ 结果非常相近, 同时与其他学者在青海湖及其 他湖泊通过地球化学示踪得到的 $F_{\mathrm{LGD}}$ 结果相近, 说明镭同位素示踪结果较为准确, 可以进一步结合营养物 质输人通量对湖泊的生物地球化学过程进行相应的评价.

\section{4 结论}

1) 通过对青海湖泉湾不同水体 ${ }^{223} \mathrm{Ra}$ 和 ${ }^{224} \mathrm{Ra}$ 的研究, 发现其 $\mathrm{Ra}$ 活度值表现为: 地下水>湖水>河水, 表层 
表 $4{ }^{224} \mathrm{Ra}$ 源、汇项计算参数

Tab.4 Calculation parameters for ${ }^{224}$ Ra sources and sinks

\begin{tabular}{|c|c|c|c|}
\hline 参数 & 物理意义 & 数值 & 单位 \\
\hline $\mathrm{V}_{\text {-lake }}$ & 湖湾平均水体积 & $9.88 \times 10^{8}$ & $\mathrm{~m}^{3}$ \\
\hline $\mathrm{H}_{\text {-lake }}$ & 湖湾平均水深 & 12 & $\mathrm{~m}$ \\
\hline $\mathrm{T}$ & 水体停留时间 & 1.8 & d \\
\hline$\lambda$ & ${ }^{224} \mathrm{Ra}$ 衰变系数 & 0.19 & $\mathrm{~s}^{-1}$ \\
\hline $\mathrm{F}_{\text {riv-w }}$ & 河流年径流量 & $1.3275 \times 10^{9}$ & $\mathrm{~m}^{3} / \mathrm{a}$ \\
\hline $\mathrm{F}_{\text {riv-s }}$ & 河流年输沙量 & $3.577 \times 10^{8}$ & $\mathrm{~kg} / \mathrm{a}$ \\
\hline $\mathrm{C}_{\text {-desorb }}$ & 河流悬浮颗粒物质量浓度 & 0.27 & $\mathrm{~kg} / \mathrm{m}^{3}$ \\
\hline$A_{- \text {desorb }}$ & ${ }^{224} \mathrm{Ra}$ 颗粒物解析活度 & 0.49 & $\mathrm{dpm} / \mathrm{g}$ \\
\hline$A_{\text {-lake }}$ & 湖水 ${ }^{224} \mathrm{Ra}$ 平均活度 & 0.504 & $\mathrm{dpm} / 100 \mathrm{~L}$ \\
\hline $\mathrm{A}_{0}$ & 最远端的 ${ }^{224} \mathrm{Ra}$ 活度 & 0.27 & $\mathrm{dpm} / 100 \mathrm{~L}$ \\
\hline $\mathrm{A}_{\mathrm{GW}}$ & 地下水 ${ }^{224} \mathrm{Ra}$ 平均活度 & 1.30 & $\mathrm{dpm} / 100 \mathrm{~L}$ \\
\hline$A_{\text {-river }}$ & 河水 ${ }^{224} \mathrm{Ra}$ 平均活度 & 0.22 & $\mathrm{dpm} / 100 \mathrm{~L}$ \\
\hline $\mathrm{F}_{\mathrm{LGD}}$ & 地下水排放通量 & / & $\mathrm{m}^{3} /\left(\mathrm{m}^{2} \cdot \mathrm{d}\right)$ \\
\hline
\end{tabular}

表 $5{ }^{224} \mathrm{Ra}$ 源汇项及 $\mathrm{F}_{\mathrm{LGD}}$ 估算

Tab.5 Sources and sinks of ${ }^{224} \mathrm{Ra}$ and estimation of $\mathrm{F}_{\mathrm{LGD}}$

\begin{tabular}{ccccc}
\hline & & 公式 & 计算结果 & 占比/\% \\
\hline 源项 & 河流输人 & $\mathrm{I}_{\text {riv }}=\mathrm{F}_{\text {riv-w }} \cdot \mathrm{A}_{\text {-river }}$ & $0.029 \times 10^{11}$ & 0.74 \\
& 悬浮颗粒物解析 & $\mathrm{I}_{\mathrm{r} \text {-desorb }}=\mathrm{F}_{\text {riv-ww }} \cdot \mathrm{C}_{\text {-desorb }} \cdot \mathrm{A}_{\text {-desorb }}$ & $1.76 \times 10^{11}$ & 44.91 \\
& 地下水输人 & $\mathrm{I}_{\mathrm{LGD}}=\mathrm{F}_{\mathrm{LGD}} \cdot \mathrm{A}_{\mathrm{GW}}$ & $2.13 \times 10^{11}$ & 54.35 \\
\hline 汇项 & 放射性衰变 & $\mathrm{I}_{\text {decay }}=\mathrm{V}_{\text {-lake }} \cdot \mathrm{A}_{\text {-lake }} \cdot \lambda$ & $3.45 \times 10^{11}$ & 88.03 \\
& 与湖水混合 & $\mathrm{I}_{\text {out }}=\left(\mathrm{A}_{\text {-lake }}-\mathrm{A}_{0}\right) \cdot \mathrm{V}_{\text {-lake }} / \mathrm{T}$ & $0.469 \times 10^{11}$ & 11.97 \\
\hline 总量 & & $\mathrm{I}_{\text {out }}+\mathrm{I}_{\text {decay }}=\mathrm{I}_{\text {riv }}+\mathrm{I}_{\mathrm{r} \text {-desorb }}+\mathrm{I}_{\mathrm{LGD}}$ & $3.919 \times 10^{11}$ & \\
\hline 单位 $\mathrm{F}_{\mathrm{LGD}}$ & & $0.54 \mathrm{~m}^{3} /\left(\mathrm{m}^{2} \cdot \mathrm{d}\right)$ & & \\
\hline
\end{tabular}

湖水、地下水和河水 ${ }^{223} \mathrm{Ra}$ 和 ${ }^{224} \mathrm{Ra}$ 的平均活度分别为 0.441 和 $0.026 \mathrm{dpm} / 100 \mathrm{~L} 、 0.061$ 和 $1.30 \mathrm{dpm} / 100 \mathrm{~L}$ 以及 0.22 和 $0.016 \mathrm{dpm} / 100 \mathrm{~L}$.

2) 在水平向上, 湖水 Ra 活度随离岸距离增加而逐 渐减小,但在河口区, 受悬浮颗粒物解析影响, 在 $4 \sim 6 \mathrm{~km}$ 左右存在随离岸距离增加 Ra 活度增大的不保守性分 布; 而垂向上, Ra 活度随湖水深度增加呈迅速减小, 但在 一定深度存在小幅增大的楔形剖面分布.

3) 通过水体滞留时间模型计算获得青海湖泉湾水 体的平均停留时间为 1.8 天, 进一步分析泉湾水体 $R a$ 的
表 6 湖泊沿岸地下水排放通量结果汇总

$$
\left(\mathrm{m}^{3} /\left(\mathrm{m}^{2} \cdot \mathrm{d}\right)\right)
$$

Tab.6 The summary results of $F_{L G D}$

of lake coast

\begin{tabular}{cc}
\hline 水量平衡模型 & 地球化学示踪 \\
\hline 青海湖 $0.18^{[21]}$ & 青海湖泉湾 $0.054 \sim 0.109$ (本文) \\
青海湖 $0.14^{[20]}$ & 青海湖西北岸 $0.05 \sim 0.1488^{[26]}$ \\
青海湖 $0.11^{[19]}$ & 巴丹吉林湖 $0.07 \sim 0.16^{[49]}$ \\
& 108 个湖泊均值 $0.074 \sim 0.148^{[50]}$ \\
\hline
\end{tabular}
源汇项, 应用镭同位素质量平衡模型获得青海湖泉湾布哈河口沿岸地下水排放通量为 $0.054 \sim 0.109 \mathrm{~m}^{3} /$ $\left(\mathrm{m}^{2} \cdot \mathrm{d}\right)$. 对比分析表明该次估算结果与通过其他方法计算出的青海湖沿岸地下水排放通量结果非常相近.

\section{5 参考文献}

[ 1 ] Burnett WC, Bokuniewicz H, Huettel M et al. Groundwater and pore water inputs to the coastal zone. Biogeochemistry, 2003, 66: 3-33.

[ 2 ] Charette MA, Sholkovitz ER. Trace element cycling in a subterranean estuary: Part 2. Geochemistry of the pore water. Geochimica et Cosmochimica Acta, 2006, 70(4) : 811-826. 
[ 3 ] Rama JF, Todd JB, Moore WS. A new method for the rapid measurement of ${ }^{224}$ Ra in natural waters. Mar Chem, 1987, 22: 43-54.

[ 4 ] Bollinger MS, Moore MS. Radium flux from a salt marsh. Nature, 1984, 309: 444-446.

[ 5 ] Levy DM, Moore WS. ${ }^{224}$ Ra in continental shelf waters. Earth and planetary Science Letters, 1985, 73: 226-230.

[ 6 ] Webster IT, Hancock GJ, Murray AS. Modelling the effect of salinity on radium desorption from sediments. Geochimica et Cosmochimica Acta, 1995, 59(12) : 2469-2476.

[ 7 ] Men W, Liu GS, Chen ZG et al. Progresses of radium isotopes applications to oceanography research. Advances in Earth Science, 2010, 25 (1) : 33-41. [门武, 刘广山, 陈志刚等. 镭同位素在海洋学研究中的应用及进展. 地球科学进展, $2010,25(1): 33-41$.]

[ 8 ] Moore WS, Arnold R. Measurement of ${ }^{223}$ Ra and ${ }^{224}$ Ra in coastal waters using a delayed coincidence counter. Journal of Geophysical Research: Oceans, 1996, 101(C1) : 1321-1329.

[ 9 ] Nozaki Y, Kasemsupaya V, Tsubota H. Mean residence time of the shelf water in the East China and the Yellow Seas determined by 228Ra/226Ra measurements. Geophysical Research Letters, 1989, 16(11) : 1297-1300.

[10] Scott MK. Moran SB. Ground water input to coastal salt ponds of southern Rhode Island estimated using ${ }^{226}$ Ra as a tracer. Journal of Environmental Radioactivity, 2001, 54: 163-174.

[11] Swarzenski PW. U/Th series radionuclides as coastal groundwater tracers. Chemical Reviews, 2007, 107(2) : 663-674.

[12] Kim G, Ryu JW, Hwang DW. Radium tracing of submarine groundwater discharge( SGD) and associated nutrient fluxes in a highly-permeable bed coastal zone, Korea. Marine Chemistry, 2008, 109: 307-317.

[13] Hwang DW, Kim G et al. The role of submarine groundwater discharge( SGD) in nutrient budgets of Gamak Bay, a shellfish farming bay, in Korea. Journal of Sea Research, 2010, 64: 224-230.

[14] Luo X, Jiao JJ, Moore WS et al. Submarine groundwater discharge estimation in an urbanizedembayment in Hong Kong via short-lived radium isotopes and its implication of nutrient loadings and primary production. Marine Pollution Bulletin, 2014, 82 : 144-154.

[15] Sun HL ed. The formation and evolution of the Qinghai-Tibet Plateau. Shanghai: Shanghai Science and Technology Press, 1996. [孙鸿烈. 青藏高原的形成演化. 上海: 上海科学技术出版社, 1996. ]

[16] Sun YL, Li XY, Tang J et al. Climate change and hydrological response in the watershed of Qinghai Lake. Resources Science, 2008, 30 (30) : 354-362. [孙永亮, 李小雁, 汤佳等. 青海湖流域气候变化及其水文效应. 资源科学, 2008, $30(30): 354-362$.

[17] Sha ZJ, Wang Q, Wang J et al. Regional environmental change and human activity over the past hundred years recorded in the sedimentary record of Lake Qinghai, China. Environmental Science and Pollution Research, 2017, 24 ( 10 ): 9662-9674.

[18] Wang Q, Sha Z, Wang J et al. Historical changes in the major and trace elements in the sedimentary records of Lake Qinghai, Qinghai-Tibet Plateau: implications for anthropogenic activities. Environmental Geochemistry and Health, 2019: 1-19.

[19] Ding YJ, Liu FJ. Estimating on water balance elements in the drainage basin of Qinghai Lake. Arid Land Geography, 1993, 16(4) : 25-30. [丁永建, 刘风景. 青海湖流域水量平衡要素的估算. 干旱区地理, 1993, 16(4): 25-30.]

[20] Yan HY, Jia SF. Water balance and water resources allocation of Qinghai Lake. J Lake Sci, 2003, 15(1) : 36-40. DOI: 10.18307/2003.0105. [燕华云, 贾绍风. 青海湖水量平衡分析与水资源优化配置研究. 湖泊科学, 2003, 15 (1): 36-40. ]

[21] Wang F, Liu J, Yan HY. Analysis on hydrological process of water balance factors in Qinghai Lake. Journal of Hydraulic Engineering, 2008, 11:1229-1238. [王芳, 刘佳, 燕华云. 青海湖水平衡要素水文过程分析. 水利学报, 2008, 11: 1229-1238.]

[22] Sha ZJ, La B, Kong FC et al. Qinghai lake gahai groundwater input and its flux. Acta Geologica Sinica, 2015, 89( S1): 282-285. [沙占江, 拉本, 孔凡翠等. 青海湖尔海地下水输人及其物质通量. 地质学报, 2015, 89(S1): 282285.]

[23] Zhao SL. Radium isotope tracer groundwater discharge flux of Gahai in Qinghai Lake[Dissertation]. Xining: Qinghai Normal University, 2014. [ 赵石否. 镭同位素示踪青海湖尔海地下水排放通量 [ 学位论文]. 西宁: 青海师范大学, 2014. ]

[24] Kong FC, Sha ZJ, Du JZ et al. Analysis of the distribution characteristics of (226) Ra and (228) Ra and their sources in the western part of Lake Qinghai. Chinese Journal of Oceanology and Limnology, 2015, 33(6) : 1402-1412.

[25] Kong FC, Sha ZJ, Luo X et al. Evaluation of lacustrine groundwater discharge and associated nutrients, trace elements and DIC loadings into Lake Qinghai in Qinghai-Tibetan Plateau, using radium isotopes and hydrological methods. Chemical Geology, 2019, 510: 31-46.

[26] Zhang K, Sha ZJ, Zhao SL et al. Radium isotope distribution in Western Qinghai Lake and its environmental significance. 
Journal of Salt Lake Research, 2015, 23(4) : 31-38. [张凯, 沙占江, 赵石否等. 青海湖西部镭同位素分布特征及环 境意义. 盐湖研究, $2015,23(4): 31-38$. ]

[27] Bian QT, Liu JQ, Luo XQ et al. Geotectonic setting, formation and evolution of the Qinghai Lake. Seismology and Geolo$g y, 2000,22(1): 20-26$. [边千蹈, 刘嘉麒, 罗小全等. 青海湖的地质构造背景及形成演化. 地震地质, 2000,22 (1) : 20-26.]

[28] Hu DS. Geological evolution of Qinghai Lake. Arid Land Geography, 1989, 12(2): 29-36. [胡东生. 青海湖的地质演 变. 干旱区地理, 1989, 12(2): 29-36.]

[29] Zhang K, Sun YG, Ju SC et al. The neotectonic process causingthe conversion of the Qinghai Lake from an outflow Lake into an Interior Lake. Remote Sensing for Land \& Resources, 2010, 86:77-81. [张焜, 孙延贵, 巨生成等. 青海湖由外 流湖转变为内陆湖的新构造过程. 国土资源遥感, 2010, 86: 77-81.]

[30] Moore WS. Sampling Radium-228 in the deep ocean. Deep-Sea Research, 1976, 23:647-651.

[31] Sun Y, Torgersen T. The effects of water content and Mn-fiber surface conditions on 224Ra measurement by 220Rn emanation. Marine Chemistry, 1998, 62:299-306.

[32] Moore WS. Oceanic concentrations of 226Ra. Earth and Planetary Science Letter, 1969, 2: 231-234.

[33] Kaufman A, Trier RM, Broecker WS et al. Distribution of 226Ra in the world ocean. Journal of Geophysical Research, 1973, 78: 8827-8848.

[34] Gonneea ME, Morris PJ, Dulaiova H et al. New perspectives on radium behavior within a subterranean estuary. Marine Chemistry, 2008, 109(3/4) : 250-267.

[35] Elsinger RJ, Moore WS. 224Ra, 228Ra, and 226Ra in Winyah Bay and Delaware Bay. Earth and Planetary Science Letters, 1983, 64(3): 430-436.

[ 36] Moore WS, Krest J. Distribution of 223Ra and 224Ra in the plumes of the Mississippi and Atchafalaya Rivers and the Gulf of Mexico. Mar Chem, 2004, 86(3) : 105-119.

[37] Chen XB, Huang YP, Xie YZ et al. Distribution of ${ }^{224}$ Ra in the Jiulong estuarine waters and its application. Acta Oceanologica Sinica, 1999, (4):54-61. [陈性保, 黄奕普, 谢永榛等. 九龙江河口区水体中 (224) Ra 的分布及其应用. 海洋学报: 中文版, 1999,(4): 54-61.]

[38 ] Xu BC, Yao QZ, Jiang XY et al. Measurement of 223Ra and 224Ra via RaDeCC and its application at Liaohe River Estuary. Marine Environmental Science, 2012, 31(2):153-158. [许博超, 姚庆祯, 江雪艳等. 辽河口水体中 ( 223) Ra、 $\sim(224)$ Ra 的 RaDeCC 法测定及应用. 海洋环境科学, 2012, 31(2) : 153-158.]

[39] Kong FC, Sha ZJ, Du JZ et al. Desorption and diffusion characteristics of radium isotopes from particles in the western part of Lake Qinghai. J Lake Sci, 2016, 28(5) : 1103-1114. DOI:10.18307/2016.0521. [孔凡翠, 沙占江, 杜金洲等. 青海 湖西岸镭同位素的解吸和扩散特征. 湖泊科学, 2016, 28(5): 1103-1114.]

[40] Beck AJ, Cochran MA. Controls on solid-solution partitioning of radium in saturated marine sands. Marine Chemistry, 2013, 156: 38-48.

[41] Jin ZD, You CF, Yu JM. Toward a geochemical mass balance of major elements in Lake Qinghai, NE Tibetan Plateau : A significant role of atmospheric deposition. Applied Geochemistry, 2009, 24: 1901-1907.

[42] Liu GS, Men W, Ji LH. Vertical mixing rate evaluation based on radium isotope distributions of Yellow Sea and East China Sea. Chinese Journal of Geophysics, 2010, 53(8): 1976-1984. [刘广山, 门武, 纪丽红. 基于镭同位素分布的黄海和 东海垂直混合速率计算. 地球物理学报, 2010, 53(8): 1976-1984.]

[43] Su N. Tracing coastal water mixing processes and submarine groundwater discharge by radium isotopes [Dissertation]. Shanghai : East China Normal University, 2013. [苏妮. 镭同位素示踪的近岸水体混合和海底地下水排泄 [ 学位论 文]. 上海: 华东师范大学, 2013.]

[44] Moore WS. Determining coastal mixing rates using radium isotopes. Continental Shelf Research, 2000, 20:1993-2007.

[45] Peterson RN, Burnett WC, Taniguchi M et al. Determination of transport rates in the Yellow River-Bohai Sea mixing zone via natural geochemical tracers. Continental Shelf Research, 2008, 19: 2700-2707.

[46] Garrison GH, Glenn CR, Mcmurtry GM. Measurement of submarine groundwater discharge in Kahana Bay, Oahu, Hawaii. Limnology and Oceanography, 2003, 48: 920-928.

[47] Taniguchi M, Burnett WC, Cable JE et al. Investigation of submarine groundwater discharge. Hydrological Processes, 2002, 16: 2115-2129.

[48 ] Taniguchi M, Iwakawa H. Submarine groundwater discharge in Osaka Bay, Japan. Limnology, 2004, (5) : 25-32.

[49] Luo X, Jiao JJ, Wang X, Liu K. Temporal 222Rn distributions to reveal groundwater discharge into desert lakes: implication of water balance in the Badain Jaran Desert, China. Hydrol, 2016, 534: 87-103.

[50] Rosenberry DO, Lewandowski J, Meinikmann K et al. Nucleation and the disregarded component in lake water and nutrient budgets. Part 1: effects of groundwater on hydrology. Hydrol Process, 2015, 29(13) : 2895-2921. 